\section{Uso universal da vacina inativada contra poliomielite}

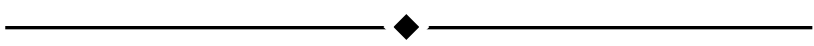

Prezado Editor,

As mudanças no cenário internacional tornam cada vez mais evidente que, na maioria dos países, o risco atual de desenvolver paralisia associada ao vírus vacinal (PAVV) ou paralisia por vírus derivados dos vírus vacinais (VdVV) é maior do que o risco de contrair paralisia por poliovírus selvagens, tornando necessária a substituição da vacina oral contra pólio (VOP) pela vacina inativada (VIP), como proposto no excelente artigo de Falleiros-Carvalho \& Weckx ${ }^{1}$.

As principais questões relacionadas à introdução da VIP nos calendários de rotina relacionam-se à indução de menor imunidade gastrintestinal, dificuldade de aplicação (uso parenteral), disponibilidade e custos. Estudos recentes revelam que crianças previamente vacinadas com duas doses da VIP apresentam baixa excreção fecal dos vírus vacinais após desafio com uma dose da VOP; além disso, o risco de reversão das cepas Sabin à neurovirulência não aumentou após o uso da VIP2,3.

As vacinas combinadas contendo VIP aumentam a aceitação da vacina inativada, pois propiciam aumento nas coberturas vacinais contra várias doenças a custos mais baixos. Nos últimos anos, aumentou o número de países que adotam a VIP em esquema seqüencial (VIP seguida por VOP) ou exclusivo; entretanto, a capacidade de produção anual da VIP é estimada em apenas 100 milhões de doses. A tecnologia para produção da VIP já é conhecida há mais de 5 décadas, porém existem poucos laboratórios credenciados para produzir essa vacina, e sua capacidade de produção é limitada ${ }^{4}$.

De acordo com a Organização Mundial da Saúde (OMS), 4 a 5 anos após a certificação de eliminação global da poliomielite, o uso da vacina oral deverá ser interrompido simultaneamente em todos os países, e os estoques de poliovírus selvagens deverão ser destruídos, mantendo-se apenas estoques de vacinas para uso oral (monovalente e trivalente) para controle de um eventual ressurgimento da doença 4 .

A interrupção da vacinação contra pólio fará com que, dentro de poucos anos, aumente o pool de suscetíveis. 0 medo do bioterrorismo torna pouco provável a interrupção da vacinação nos países desenvolvidos que já adotam a VIP. Vale lembrar que, para a produção dessa vacina, são necessários grandes estoques de vírus selvagens e, por maior que seja o controle internacional sobre os laboratórios produtores, é impossível impedir futuros riscos de acidentes ou de ações criminosas, com a reintrodução dos vírus selvagens em populações não-imunes ${ }^{1,4}$. Por esses motivos, estão sendo desenvolvidas novas vacinas inativadas, produzidas a partir das cepas Sabin. Além disso, já estão em discussão as formas de repasse dessa tecnologia para países em desenvolvimento ${ }^{5}$.

Enquanto as novas vacinas inativadas não estiverem disponíveis, os países industrializados estarão livres da pólio se mantiverem altas coberturas com a VIP, restando discutir a política para países que atualmente utilizam exclusivamente a VOP, como o Brasil. Se a vacinação com a VOP for interrompida sem que se consiga introduzir a VIP, em pouco tempo a população desses países se tornará suscetível à pólio e dependerá da eficácia da vigilância epidemiológica para detectar precocemente os casos da doença e dos estoques da vacina VOP para controlar eventuais surtos da doença. Vale lembrar que os vírus derivados de vírus vacinais têm circulado por muitos anos antes de sua detecção e que, após a confirmação de casos de paralisia por VdVV, o tempo médio para organizar as campanhas de vacinação em massa para evitar a disseminação desses vírus tem sido superior a 6 meses $^{6}$. Portanto, é necessário intensificar a vigilância epidemiológica, que infelizmente está abaixo dos padrões recomendados pela OMS em diversos países, incluindo o Brasil.

Como os principais obstáculos atuais à erradicação da pólio estão relacionados aos VdVV (circulantes e excretados por imunodeficientes) ${ }^{7-9}$, é essencial providenciar rapidamente a adoção de novas estratégias de vacinação. Assim como Falleiros-Carvalho \& Weckx, acreditamos ser necessário o planejamento para introdução da VIP em nosso país, assim como a busca de medidas que viabilizem a produção local dessa vacina para evitar a dependência externa.

Em 2005-2006, apenas quatro países permanecem endêmicos para os poliovírus selvagens (Nigéria, Índia, Paquistão e Afeganistão); entretanto, desde 2003, 22 países anteriormente livres da pólio foram reinfectados, devido à queda nas coberturas vacinais ${ }^{9}$. Tanto a VOP como a VIP são efetivas na prevenção da poliomielite, mas nenhuma dessas vacinas evita totalmente a replicação silenciosa dos vírus selvagens e VdVV no intestino. A transmissão dos poliovírus só pode ser evitada através de elevadas coberturas vacinais. Até que se consiga implementar o uso da VIP em todo o país, a vacinação com a VOP deve ser mantida para evitar o risco de paralisia por vírus selvagens importados de outros países ou causada por VdVV.

\section{Referências}

1. Falleiros-Carvalho LH, Weckx LY. Universal use of inactivated polio vaccine. J Pediatr (Rio J). 2006;82(3 Suppl):S75-82.

2. Laassri $M$, Lottenbach $K$, Belshe R, Wolff $M$, Rennels $M$, Plotkin $\mathrm{S}$, et al. Effect of different vaccination schedules on excretion of oral poliovirus vaccine strains. J Infect Dis. 2005;192:2092-8. 
3. Laassri $M$, Lottenbach $K$, Belshe $R$, Rennels $M$, Plotkin $S$, Chumakov K. Analysis of reversions in the 5 '-untranslated region of attenuated poliovirus after sequential administration of inactivated and oral poliovirus vaccines. J Infect Dis. 2006;193:1344-9.

4. Heymann DL, Aylward RB. The polio eradication endgame. As polio eradication nears realization, such real-world vaccination strategies could hold lessons for the future in AIDS vaccine development. IAVI Rep. 2006;10:13-7.

5. Kreeftenberg $H$, van der Velden $T$, Kersten $G$, van der Heuvel N, de Bruijn M. Technology transfer of Sabin-IPV to new developing country markets. Biologicals. 2006;34:155-8.

6. Agol VI. Vaccine-derived polioviruses. Biologicals 2006; 34: 103-8.

7. Martin J. Vaccine-derived poliovirus from long term excretors and the end game of polio eradication. Biologicals. 2006;34: 117-22.

8. Performance of acute flaccid paralysis (AFP) surveillance and incidence of poliomyelitis 2004-2005. (Data received in WHO headquarters as of 7 March 2006). Wkly Epidemiol Rec. 2006;81: 114-5.

9. Aylward RB, Maher C. Interrupting poliovirus transmission - new solutions to an old problem. Biologicals. 2006;34:133-9.

doi:10.2223/JPED.1574

\section{Lucia Ferro Bricks}

Professora Doutora, Departamento de Pediatria, Faculdade de Medicina, Universidade de São Paulo (USP), São Paulo, SP. Membro, Comissão Permanente de Assessoramento em Imunizações, Secretaria de Estado da Saúde do Estado de São Paulo, São Paulo, SP.Ifbricks@gmail.com

Conflitos de interesse: nenhum para este texto. Já participou como investigadora de estudos multicêntricos sobre vacinas financiados pelos laboratórios Whyeth e Merck Sharp Dohme e recebeu grants por palestras sobre imunizações em congressos e jornadas científicas patrocinadas pelos laboratórios Whyeth, Merck, Sanofipasteur e GSK.

\section{Efetividade da terapia dupla e tríplice contra o HIV}

\section{Prezado Editor,}

Gostaria de tecer algumas considerações e comentários sobre o artigo original de Romanelli et al. ${ }^{1}$ e sobre o editorial de Oleske².

Primeiramente, torna-se mandatório destacar o grande valor do estudo de Romanelli ${ }^{1}$ no sentido de fornecer informações importantes para o manejo da infecção pelo HIV/AIDS em crianças. Este e outros estudos respaldam a mudança de conduta nos guias de tratamento de infecção pelo HIV em crianças ao longo dos últimos anos. Conduta esta que passou por fases de indicação formal de terapia dupla inicial, de fase seguinte de restrição de indicação de terapia dupla (casos leves) até a fase atual com indicação formal de terapia tripla inicial.

Em relação ao editorial de Oleske ${ }^{2}$, torna-se necessário adicionar alguns esclarecimentos e comentários. No final do primeiro parágrafo, Oleske afirma que a patogênese da infecção pelo HIV e que os princípios gerais do tratamento são os mesmos para adultos, adolescentes, crianças e bebês.
Contudo, já está muito bem definido que a dinâmica viral e a imunopatogênese da infecção pelo HIV em adultos e crianças apresentam diferenças significativas, sendo que algumas delas ainda precisam ser elucidadas ${ }^{3,4}$. Podemos, ainda, acrescentar que é exatamente a diferença na dinâmica viral e patogênese da infecção pelo HIV em crianças que norteia as indicações diferentes dos guias de terapia anti-retroviral em crianças e adultos, principalmente em relação aos parâmetros para início de tratamento, parâmetros de sucesso e falha terapêutica e características peculiares da reconstituição imunológica em crianças 5,6 .

No segundo parágrafo, Oleske afirma que a progressão mais rápida da doença em crianças é conseqüência da farmacocinética das múltiplas drogas usadas no tratamento do HIV. Sabemos que, quando falamos em progressão da infecção pelo HIV, geralmente estamos descrevendo aspectos da história natural da infecção, que, para ser natural, requer ausência de terapia anti-retroviral. Não cabe, desse modo, esse tipo de inferência ou relação casual. Também senti falta, no texto, de referência à escassez de estudos de farmacocinética em crianças, principalmente nos primeiros 6 meses de vida. Os estudos existentes geralmente apresentam casuística muito pequena e de faixa etária variada 7,8 .

Finalmente, gostaria de lembrar aos pediatras e infectologistas que assistem crianças com infecção pelo HIV/AIDS que o manejo da infecção pelo HIV em crianças no Brasil deve ser norteado pelo Guia de Tratamento Clínico da Infecção pelo HIV em Crianças, elaborado e distribuído pelo Programa Nacional de DST/AIDS do Ministério da Saúde. Esse documento é atualizado periodicamente, e sua versão 2006 já está disponível no site do Programa (www.aids.gov.br).

\section{Referências}

1. Romanelli RM, Pinto JA, Melo LJ, Vasconcelos MA, Pereira Rde M. Effectiveness of dual and triple antiretroviral therapy in the treatment of HIV-infected children. J Pediatr (Rio J). 2006;82: 260-5.

2. Oleske JM. When should we treat children with HIV? J Pediatr (Rio J). 2006;82:243-5.

3. Tiemessen CT, Kuhn L. Immune pathogenesis of pediatric HIV1 infection. Curr HIV/AIDS Rep. 2006;3:13-9.

4. Chakraborty R. HIV-1 infection in children: a clinical and immunologic overview. Curr HIV Res. 2005;3:31-41.

5. Resino S, Seoane E, Gutierrez MD, Leon JA, Munoz-Fernandez MA. CD4(+) T-cell immunodeficiency is more dependent on immune activation than viral load in HIV-infected children on highly active antiretroviral therapy. J Acquir Immune Defic Syndr. 2006;42:269-76.

6. Zanchetta M, Walker S, Burighel N, Bellanova D, Rampon O, Giaquinto $C$, et al. Long-term decay of the HIV-1 reservoir in HIV-1-infected children treated with highly active antiretroviral therapy. J Infect Dis. 2006;193:1718-27. Epub 2006 May 10.

7. Fraaij PL, van Kampen JJ, Burger DM, de Groot R. Pharmacokinetics of antiretroviral therapy in HIV-1-infected children. Clin Pharmacokinet. 2005;44:935-56.

8. King JR, Kimberlin DW, Aldrovandi GM, Acosta EP. Antiretroviral pharmacokinetics in the paediatric population: a review. Clin Pharmacokinet. 2002;4:1115-33.

doi:10.2223/JPED.1575

\section{Edvaldo Souza}

Mestre, médico, professor e pesquisador, Instituto Materno-Infantil Professor Fernando Figueira (IMIP), Recife, PE. Membro, Comitê Assessor para Terapia Anti-Retroviral em Crianças Infectadas pelo HIV, Programa Nacional de DST e AIDS, Ministério da Saúde, Brasil. essouza@terra.com.br, edsouza@imip.org.br 